\title{
SEGURANÇA ALIMENTAR E NUTRICIONAL EM GESTANTES E SUA ASSOCIAÇÃO COM FATORES DE RISCO PARA PRÉ-ECLÂMPSIA
}

\section{FOOD AND NUTRITIONAL SECURITY IN PREGNANT WOMEN AND ITS ASSOCIATION WITH RISK FACTORS FOR PRE-ECLAMPSIA}

\begin{abstract}
Bárbara Gomes Santos Silva ${ }^{1} *$ Karla Polyana de Moura Guimarães Souza ${ }^{2} *$ Maria Luziene de Sousa $^{2}$ Gomes $^{3} *$ Laiara de Alencar Oliveira ${ }^{4}$ Nadya dos Santos Moura ${ }^{5} *$ Dafne Paiva Rodrigues $^{6} *$ Mônica Oliveira Batista Oriá
\end{abstract}

\section{RESUMO}

Objetivo: Associar a insegurança alimentar com os fatores de risco para desenvolvimento da Pré-Eclâmpsia. Métodos: Estudo transversal, envolvendo gestantes cadastradas em cinco unidades da estratégia de saúde da família. Para a coleta de dados foram utilizados um formulário estruturado para caracterização das participantes, o Questionário de Frequência Alimentar e a Escala Brasileira de Insegurança Alimentar. Foram conduzidas análises descritiva, bivariada e o Teste Qui-quadrado de Pearson. Resultados: O estudo envolveu 44 gestantes com média de idade de 26,33 anos (Desvio padrão $= \pm 6,5$ ). Quanto a classificação da insegurança alimentar das gestantes, $(\mathrm{N}=34,75,6 \%)$ estavam em situação de segurança alimentar. Os dados apontam que a insegurança alimentar foi associada ao fator de risco "história familiar de pré-eclâmpsia", obtendo associação $(\mathrm{p}=0,036)$. Conclusão: $\mathrm{O}$ estudo demonstrou a associação entre a insegurança alimentar e os fatores de risco para desenvolvimento da PE em gestantes. Torna-se necessário que os profissionais de saúde atentem-se para a existência dessas condições durante o período gestacional. Deve-se estimular estudos que utilizem instrumentos como o QFA e a EBIA, pois a partir deles é possível identificar a insegurança alimentar em gestantes.

Descritores: Segurança Alimentar e Nutricional; Fatores de Risco; Pré-eclâmpsia; Gestantes; Promoção da Saúde.

\begin{abstract}
Objective: Associate food insecurity with risk factors for the development of Pre-Eclampsia. Methods: Cross-sectional study, involving pregnant women registered in five units of the family health strategy. For data collection, a structured form to characterize the participants, the Food Frequency Questionnaire and the Brazilian Food Insecurity Scale were used. Descriptive and bivariate analyzes and Pearson\&\#39;s Chisquare test were conducted. Results: The study involved 44 pregnant women with a mean age of 26.33 years (standard deviation $= \pm 6.5)$. As for the classification of food insecurity of pregnant women, $(\mathrm{N}=34,75.6 \%)$ were in a situation of food security. The data indicate that food insecurity was associated with the risk factor "family history of pre-eclampsia", obtaining an association $(\mathrm{p}=0.036)$. Conclusion: The study demonstrated the association between food insecurity and risk factors for the development of PE in pregnant women. It is necessary that health professionals pay attention to the existence of these conditions during the gestational period. Studies that use instruments such as the FFQ and the EBIA should be encouraged, as they make it possible to identify food insecurity in pregnant women.
\end{abstract}

Keywords: Food and Nutrition Security; Risk Factors; Pre-Eclampsia; Pregnant. Women; Health Promotion.

\footnotetext{
${ }^{1}$ Enfermeira, mestranda no Programa de Pós-graduação em Enfermagem, Universidade Federal do Ceará - UFC. Fortaleza, Ceará, Brasil. ID Orcid: 0000-0001-8892-2293

${ }^{2}$ Enfermeira, Universidade Federal do Piauí - UFPI. Picos, Piauí, Brasil. ID Orcid: 0000-0001-9691-5712

${ }^{3}$ Enfermeira, doutoranda no Programa de Pós-graduação em Enfermagem, Universidade Federal do Ceará - UFC. Fortaleza, Ceará, Brasil. ID Orcid: 0000-0001-8583-0959

${ }^{4}$ Graduanda em Enfermagem, Universidade Federal do Piauí - UFPI. Picos, Piauí, Brasil. ID Orcid: 0000-0002-3653-1108

${ }^{5}$ Enfermeira, Professora Assistente, Universidade Federal do Piauí - UFPI. Picos, Piauí, Brasil. ID Orcid: 0000-0001-5081-5206

${ }^{6}$ Enfermeira, Professora Associada. Programa de Pós-graduação em Cuidados Clínicos em Enfermagem e Saúde, Universidade Estadual do Ceará - UECE. Fortaleza, Ceará, Brasil. ID Orcid: 0000-0001-8686-3496

${ }^{7}$ Enfermeira, Professora Associada. Programa de Pós-graduação em Enfermagem, Universidade Federal do Ceará - UFC. Fortaleza, Ceará, Brasil. ID Orcid: 0000-0002 1483-6656
} 


\section{INTRODUÇÃO}

A gestação é um período em que ocorrem muitas transformações no corpo da mulher, demandando assim cuidados maiores com a saúde materna, especialmente no que concerne em uma boa alimentação, auxiliando, assim, na sua saúde, bem como no crescimento e desenvolvimento fetal. A alimentação inadequada, nesse período, pode favorecer o desenvolvimento de patologias ou agravar condições de saúde já existentes.

Nesse sentido, a Política Nacional de Promoção da Saúde estabelece a alimentação adequada e saudável como um tema prioritário, buscando promover ações relativas à alimentação adequada e saudável, visando alcançar a promoção da saúde e a segurança alimentar e nutricional, contribuindo com as ações e com as metas de redução da pobreza, com a inclusão social e com a garantia do direito humano à alimentação adequada e saudável ${ }^{(1)}$.

Nessa perspectiva, a segurança alimentar consiste no direito de todos ao acesso regular e permanente a alimentos de qualidade, em quantidade suficiente, sem comprometer o acesso a outras necessidades essenciais, tendo como base práticas alimentares promotoras de saúde que respeitem a diversidade cultural e que sejam ambiental, cultural, econômica e socialmente sustentáveis $^{(2)}$. Contudo, a Insegurança Alimentar (IA), configura-se como oposto dessa situação. A IA também tem sido considerada um fator de risco para a saúde da gestante, há evidências que associam a IA ao comprometimento de seu estado nutricional, aumento da pressão arterial, hiperglicemia, maior prevalência de anemia e repercussões na saúde do binômio mãe e filho ${ }^{(3)}$.

No tocante as complicações que podem acometer a gestação, a Pré-Eclâmpsia (PE) é uma síndrome caracterizada por hipertensão arterial identificada, pela primeira vez, após a $20^{\mathrm{a}}$ semana associada à proteinúria e pode se sobrepor a outro estado hipertensivo desaparecendo até a $12^{\mathrm{a}}$ semana após o parto $^{(4)}$. O desenvolvimento de tal síndrome pode ser motivado por fatores modificáveis, como a obesidade, as dietas hipoprotéicas e hipersódicas $^{(5)}$ que em geral podem ser evitadas ao melhorar a nutrição materna. Assim, torna-se indispensável a adoção de hábitos alimentares saudáveis, pois o estado nutricional na gestação representa um fator importante para a prevenção do desenvolvimento de $\mathrm{PE}^{(6)}$.

\section{O American College Of Obstetricians} And Gynecologists (ACOG) considera como fatores de risco para PE: primiparidade, préeclâmpsia prévia, hipertensão crônica, doença renal crônica ou ambas, história de trombofilia, gestação múltipla, fertilização in vitro, história familiar de pré-eclâmpsia, diabetes mellitus tipo 1 e 2, obesidade, lupús eritematoso sistêmico, idade materna avançada (> 40 anos). Dito isto, torna-se fundamental que o enfermeiro no âmbito da 
Atenção Primária em Saúde (APS) identifique gestantes através desses fatores e forneça assistência adequada em tempo oportuno ${ }^{(7)}$.

No Brasil, são incipientes os estudos que envolvam a IA em gestantes ${ }^{(3)}$, sobretudo associada a PE. Tendo em vista que a PE é uma das principais causas de morte materna e fetal, afetando 3 e $5 \%$ das gravidezes do mundo $^{(8)}$ e que no Brasil, representa a primeira causa de morte materna ${ }^{(9)}$. Considera-se essencial a investigação fatores que possam estar associados a doença. Para tanto, este estudo objetivou associar a IA com os fatores de risco para desenvolvimento da PE.

\section{MÉTODOS}

Trata-se de um estudo transversal, realizado no período de agosto a novembro de 2018, em cinco unidades da Estratégia Saúde da Família que apresentavam o maior quantitativo de gestantes cadastradas em consulta de pré-natal no SISPRENATAL (Sistema de Monitoramento e Avaliação do Pré-Natal, Parto, Puerpério e Criança) em um município do interior do estado do Piauí.

A população de gestantes cadastradas nas consultas de pré-natal nas cinco unidades da ESF era de 98 mulheres. A seleção da amostra aconteceu de forma não probabilística, respeitando os seguintes critérios de inclusão: gestantes cadastradas e acompanhadas nas equipes da ESF da zona urbana até a $12^{\mathrm{a}}$ semana gestacional (recomendação indica que o melhor período para rastreamento da PE é no primeiro trimestre gestacional) ${ }^{(10)}$ que estivessem presentes nos dias das consultas de pré-natal na unidade da ESF. Foram adotados como critérios de exclusão: gestantes que apresentavam PE na gestação atual e as que estavam com mais de 12 semanas gestacionais. Após os critérios de exclusão e recusas, a amostra final resultou em um total de 44 gestantes.

Os dados foram coletados por meio de entrevista norteada por três instrumentos em sala reservada na ESF. O primeiro foi um formulário estruturado e preenchido pela pesquisadora responsável, contendo variáveis sociodemográficas (idade, escolaridade, ocupação, renda, cor da pele autorreferida); características maternas (idade gestacional, quantidade de partos, quantidade de abortos, uso de álcool e outras drogas, antecedentes clínicos e familiares, uso de suplementação); como também fatores de risco para desenvolvimento da PE, utilizou-se: primiparidade, pré-eclâmpsia prévia, hipertensão arterial, gestação múltipla, história familiar de pré-eclâmpsia, obesidade e idade materna avançada (> 40 anos) $)^{(7)}$.

Os outros dois instrumentos utilizados foram o Questionário de Frequência Alimentar (QFA) $)^{(11)}$ e a Escala Brasileira de Insegurança Alimentar (EBIA) ${ }^{(12)}$ que foram preenchidos pelas gestantes. 
O QFA é um instrumento que objetiva a avaliação da dieta habitual de grupos populacionais. Os grupos de alimentos constantes no questionário são: sopas e massas, carnes e peixes, leite e derivados, leguminosas e ovos, arroz e tubérculos, verduras e legumes, molhos e temperos, frutas, bebidas, pães e biscoitos, e por fim, doces e sobremesas. Para cada grupo alimentar foi questionado com que frequência a gestante costumava comer cada item, a gradação variava de nunca ou raramente comeu, para comeu de 1 a 10 vezes no último ano, e, a respectiva unidade de tempo (dia, semana, mês ou ano).

Solicitou-se as respostas para o tamanho da porção individual usual em relação a porção média indicada, sendo graduada em $\mathrm{P}=$ menor que a porção média, $\mathrm{M}=$ igual a porção média, $\mathrm{G}=$ maior que a porção média e $\mathrm{E}=$ bem maior que a porção média. A quantidade das poções foi demonstrada por meio de exemplos como: um prato, uma concha, uma colher, uma fatia, um copo ou unidade de determinado alimento. As gestantes foram esclarecidas a escolher somente um item para cada opção, alguns dos grupos de alimentos possuíam exemplos de alimentos para facilitar o preenchimento. As gestantes foram recomendadas a não deixarem itens em branco.

A EBIA é uma escala composta por 15 questões quando houver a presença de sujeitos menores de 18 anos e 8 questões quando não tem presença de menores de 18 anos. As respostas da EBIA são: sim ou não, de modo que cada resposta afirmativa corresponde a 1 ponto, que avaliam a percepção e a experiência com a fome dos residentes de um domicílio nos últimos três meses. A pontuação da EBIA segue a classificação de acordo com os pontos de corte descritos no quadro a seguir.

Quadro 1 - Pontuação para classificação dos domicílios com e sem menores 18 anos de idade.

\begin{tabular}{|l|l|l|}
\cline { 2 - 3 } \multicolumn{1}{c|}{} & \multicolumn{2}{l|}{ Pontos de corte para domicílios } \\
\hline Classificação & Com menores de 18 anos & Sem menores de 18 anos \\
\hline Segurança Alimentar & 0 & 0 \\
Insegurança Alimentar leve & $1-5$ & $1-3$ \\
Insegurança Alimentar moderada & $6-9$ & $4-5$ \\
Insegurança Alimentar grave & $10-14$ & $6-8$ \\
\hline
\end{tabular}

Fonte: Instituto Brasileiro de Geografia e Estatística. Pesquisa Nacional por Amostra de Domicílios, 2010. 
Os dados foram processados no programa Statistical Package for the Social Sciences (SPSS) versão 20.0, foram realizados testes de estatística descritiva mediante frequências absolutas e relativas, desvio padrão e média. E, ainda, realizaramse análises bivariadas utilizando teste quiquadrado de Pearson, considerando o nível de significância $\mathrm{p} \leq 0,05$, cujos dados estão apresentados em tabelas.

Esta pesquisa foi aprovada pelo Comitê de Ética em Pesquisa da Universidade Federal do Piauí, conforme parecer $\mathrm{n}^{\circ}$ 2.838.357/2018, foram seguidos os princípios éticos contidos na Resolução $\mathrm{n}^{\circ}$ 466/12 do Conselho Nacional de Saúde que rege pesquisas envolvendo seres humanos. As participantes foram esclarecidas sobre a pesquisa e assinaram os termos de Consentimento ou Assentimento (gestantes menores de 18 anos) em duas vias, assegurando sua participação, bem como o anonimato e o sigilo.

\section{RESULTADOS}

O estudo envolveu 44 gestantes, a média de idade foi de 26,33 (Desvio padrão = ₫6,5), com idade mínima de 17 e a máxima 42 anos. Em relação à escolaridade $80 \%$ das gestantes possuíam menos de 9 anos de estudo, quanto à ocupação 20 (44,4\%) eram donas de casa. A renda pessoal predominante foi inferior a um salário mínimo $(n=17$,
$37,8 \%$ ) e a cor da pele autorreferida predominante foi a parda $(\mathrm{n}=26,57,8 \%)$.

Quanto a caracterização do perfil obstétrico, a maior parte delas estava no segundo trimestre de gestação $(n=16,35,6 \%)$, e eram multíparas $(n=26,57,8 \%)$. Das participantes, seis $(13,3 \%)$ já tiveram pelo menos um aborto $(0,18 \pm 0,4), 16(35,6 \%)$ infecção urinária e seis $(13,3 \%)$ apresentaram alteração glicêmica pelo menos uma vez na vida. Em relação à prática de hábitos nocivos à saúde três $(6,7 \%)$ relataram alcoolismo e uma $(2,2 \%)$ relatou fazer uso de drogas ilícitas.

No que se refere ao comportamento alimentar, de acordo com o QFA, no grupo sopas e massas, o alimento mais consumido foi pizza ou panqueca $(n=33,73,3 \%)$, com um consumo de 1 a 4 vezes por semana $(n=23$, $51,1 \%$ ), geralmente em uma porção tamanho média $(\mathrm{n}=17,37,8 \%)$. No grupo carnes e peixes, o alimento mais consumido foi a carne bovina, miúdos ou vísceras $(\mathrm{n}=39,86,7 \%)$, consumidos de 1 a 4 vezes por dia $(n=14$, $31,1 \%)$, em uma porção média $(n=24,53,3 \%)$. No grupo leite e derivados, o leite foi o mais consumido ( $\mathrm{n}=33,73,3 \%)$, de 1 a 4 vezes por semana $(n=17,37,8 \%)$ em porção média $(\mathrm{n}=23,51,1 \%)$.

No grupo leguminosas e ovos, os ovos $(\mathrm{n}=39,86,7 \%)$ foram consumidos numa frequência de 1 a 4 vezes por semana $(n=32$, $71,1 \%)$ em porção média $(n=22,48,9 \%)$ e a leguminosa mais consumida foi o feijão 
(n=32, 71,1\%), com uma frequência de 1 a 4 vezes por dia $(n=27,60,0 \%)$ em porção média $(n=21,46,7 \%)$.

No grupo arroz e tubérculos, o alimento mais consumido foi o arroz $(n=33,73,3 \%)$, com uma frequência de 1 a 4 vezes por dia $(\mathrm{n}=32,71,1 \%)$ em porção média $(\mathrm{n}=24$, $53,3 \%)$. No grupo verduras e legumes, o alimento mais consumido foi o tomate $(n=34$, $75,6 \%)$, de 1 a 4 vezes por dia $(n=20,44,4 \%)$ em porção pequena $(n=21,46,7 \%)$. Em relação ao grupo molhos e temperos, o alimento mais consumido foi o óleo ou azeite $(n=24,53,3 \%)$, em uma frequência de 1 a 4 vezes por semana $(n=12,26,7 \%)$, em porção média $(\mathrm{n}=14,31,1 \%)$.

No grupo frutas, a mais consumida foi a banana $(n=34,75,6 \%)$, numa frequência de 1 a 4 vezes por dia $(n=21,46,7 \%)$ numa porção média $(\mathrm{n}=26,57,8 \%)$. No grupo bebidas, a mais consumida foi o suco natural $(n=32$, 71,1\%), em uma frequência de 1 a 4 vezes por dia $(n=21,46,7 \%)$, em uma porção pequena ou média $(\mathrm{n}=16,35,6 \%)$.

No grupo pães e biscoitos, o alimento mais consumido foi o pão de forma $(n=39$, $86,7 \%$ ), numa frequência de 1 a 4 vezes por semana $(n=29,64,4 \%)$ em porção pequena $(n=20,44,4 \%)$. No grupo doces e sobremesas, os alimentos mais consumidos foram, tortas e pudins ( $\mathrm{n}=29,64,4 \%)$, com uma frequência de 1 a 4 vezes por semana $(n=15,33,3 \%)$ numa porção média $(n=14,31,1 \%)$.

Quanto a suplementação alimentar $84,4 \%$ gestantes afirmaram realizar, sendo citadas as suplementações de sulfato ferroso $(\mathrm{n}=12,26,7 \%)$ e ácido fólico $(\mathrm{n}=10,22,2 \%)$.

A classificação da insegurança alimentar das gestantes de acordo com a EBIA, está disposta na Tabela 1.

Tabela 1 - Classificação da insegurança alimentar das gestantes conforme a Escala Brasileira de Insegurança Alimentar. Picos, Piauí, Brasil, 2018 (n=44).

\begin{tabular}{lc}
\hline \multicolumn{1}{c}{ Variáveis } & N\% \\
\hline Segurança Alimentar & $34(75,60)$ \\
Insegurança Alimentar Leve & $6(13,30)$ \\
Insegurança Alimentar Moderada & $3(6,70)$ \\
Insegurança Alimentar Grave & $1(2,20)$ \\
\hline
\end{tabular}

A Tabela 2 representa a relação entre os fatores de risco para pré-eclâmpsia presentes nas gestantes e IA. 
Tabela 2 - Relação entre fatores de risco para Pré-eclâmpsia e Insegurança Alimentar das gestantes. Picos, Piauí, Brasil, 2018 (n=44).

\section{Escala Brasileira de Insegurança Alimentar}

\begin{tabular}{llllll}
\hline Fatores de Risco para & Segurança & Insegurança & Insegurança & Insegurança & $p^{* * *}$ \\
Pré-eclâmpsia & Alimentar & Alimentar & Alimentar & Alimentar & \\
& & Leve & Moderada & Grave &
\end{tabular}

Primiparidade

$\operatorname{Sim}$

21

3

0,610

Não

3

1

1

(

Pré-eclâmpsia Prévia

Sim

Não

1

1

0

0

0,489

Hipertensão Arterial

Sim

Não

5

3

1

Gestação Múltipla

Sim

Não 34

5

3

1

História Familiar de

Pré-eclâmpsia

Sim

Não

$$
7
$$

$$
0
$$$$
6
$$

2

1

Obesidade

Sim

$$
6
$$

28

0

1

0

0,556

Não

\section{Idade Materna}

Avançada

Sim

6

2

1
0,893

Não 32

0 6

$\begin{array}{ll}0 & 0 \\ 3 & 1\end{array}$

*Teste Qui-quadrado de Pearson. **Valor de $p$ nível de significância.

O dado referente ao fator de risco "história familiar de pré-eclâmpsia" apresentou associação com a insegurança alimentar $(\mathrm{p}=0,036)$. 


\section{DISCUSSÃO}

Este estudo apresenta limitações. Por se tratar de um estudo transversal, não houve estabelecimento de relações de causa e efeito, assim como não houve a possibilidade do acompanhamento dos hábitos alimentares a longo prazo, bem como a avaliação do desenvolvimento da PE nas gestantes. Além disso, aponta-se que a pesquisa foi realizada em cinco ESF, que constituem serviços públicos de saúde, com gestantes atendidas no pré-natal de baixo risco, ocasionando a redução do quantitativo, assim como a homogeneidade da amostra.

Contudo, esta pesquisa contribuiu com informações sobre hábitos alimentares e IA em gestantes, bem como sua associação com a PE, que conta com dados escassos na literatura nacional e internacional, e, com isso, forneceu informações para o desenvolvimento de estímulo a hábitos alimentares saudáveis, principalmente durante a gestação, que pode resultar em melhores desfechos maternos e perinatais. Realizar estudos como esse, em um país em desenvolvimento pode oferecer evidências para o direcionamento de políticas públicas, considerando que a maioria dos estudos que abordam essa temática são realizados em países desenvolvidos ${ }^{(13)}$ e não oferecem um parâmetro que possa ser generalizado.

No que se refere à média de idade encontrada para as gestantes estudadas, os dados encontrados vão ao encontro dos demais estudos sobre IA em gestantes, que constatam uma prevalência de mulheres jovens, na faixa etária de 24 anos, com idade mínima e máxima de 14 e 44 anos, respectivamente, apresentavam renda familiar mensal maior que um salário mínimo e mais de quatro anos de estudo ${ }^{(3)}$. Estudos demonstram a relação da IA com menor renda familiar, baixa escolaridade, maior número de moradores no domicílio, condições inadequadas de saneamento básico, ausência de vínculo empregatício ${ }^{(14-15)}$.

Quanto a frequência alimentar, observou-se nessa pesquisa um padrão de consumo alimentar denominado "tradicional", justamente por ser representado por alimentos que fazem parte da rotina alimentar dos brasileiros, visto o consumo diário de arroz, feijão e pães por grande parte das gestantes avaliadas, o que demonstra que essa prática de consumo ainda compõe a alimentação cotidiana, com uma frequência mínima de pelo menos uma vez ao dia ${ }^{(16)}$. A alimentação adequada para as mulheres, principalmente durante a fase gestacional, é um fator crítico para a melhoria da saúde e da qualidade de vida materna e fetal ${ }^{(14)}$.

No que concerne a suplementação alimentar, a maioria das gestantes afirmaram realizar, sendo citadas as suplementações de sulfato ferroso e ácido fólico. Uma Revisão Sistemática afirmou que a suplementação com sulfato ferroso e ácido fólico podem ter uma relação positiva e benéfica frente a 
possibilidade de desenvolvimento da PE. No entanto, são evidências muito heterogêneas, que não permitem recomendações totalmente eficazes como tratamento preventivo. Apesar disso, salienta-se que o estado nutricional prégestacional é um fator importante para o desenvolvimento de PE e que os efeitos da suplementação são potencializados quando realizados no início de gestação ${ }^{(17)}$.

A maioria das gestantes desse estudo apresentavam-se em condição de segurança alimentar, divergindo de um estudo realizado no Nordeste brasileiro, em que a maioria da amostra encontrava-se em situação de $\mathrm{IA}^{(3)}$. A IA é considerada um problema de saúde mundial e um fenômeno complexo determinado por múltiplos fatores e causas, portanto, a situação alimentar de uma dada população apresenta variáveis distintas, que englobam a situação econômica, psicossocial, política, cultural e étnica ${ }^{(15)}$. Destaca-se que os padrões alimentares são muito específicos e por vezes são definidos por padrões geográficos e regionais.

Os resultados de um estudo realizado na Ásia mostraram que a insegurança alimentar aumentou significativamente a probabilidade de complicações na gravidez. $\mathrm{O}$ aumento da chance de hipertensão induzida pela gravidez, pré-eclâmpsia, anemia e diabetes durante a gravidez foi visivelmente significativo no grupo com insegurança alimentar, o que foi estatisticamente significativo apenas para pré-eclâmpsia. A maior incidência foi observada na prevalência de pré-eclâmpsia, que era quase quatro vezes maior $^{(18)}$. Ressaltando a necessidade de maiores investigações sobre a relação da IA e a PE.

No que tange a associação entre a insegurança alimentar e os fatores de risco para PE, a variável história familiar de préeclâmpsia apresentou significância estatística. Resultados de uma pesquisa semelhante realizada em uma capital do Nordeste encontraram relação estatística entre PA e algumas variáveis obstétricas como história familiar para $\mathrm{PE}$ e história pessoal para $\mathrm{PE}^{(19)}$. Estes resultados, se comparados com os obtidos neste estudo levam à observação de que, se as variáveis que relacionam-se com a IA são as mesmas que relacionam-se com a $\mathrm{PE}$, o cuidado relacionado à segurança alimentar para gestantes que possuem esses fatores de risco deve ser intensificado, a fim de promover um menor risco para essas gestantes.

Uma Revisão Sistemática evidenciou que nos últimos anos, o número de estudos publicados sobre as condições de segurança alimentar e nutricional em mulheres grávidas aumentou. Toda via, essa produção científica concentrou-se principalmente nos países da América do Norte. Fica, portanto, evidente a imprescindibilidade de realização de estudos sobre o tema em países com contextos sociais e epidemiológicos diversos, considerando os diversos desfechos negativos para a saúde da 
mulher associados à insegurança alimentar e nutricional, principalmente no período gestacional $^{(14)}$.

Nesse contexto, os enfermeiros representam um papel fundamental no cuidado a mulheres em risco de saúde ${ }^{(20)}$. Tendo em vista que tanto a PE como a IA caracterizam-se por serem dois problemas de saúde pública e que podem evoluir para desfechos desfavoráveis, torna-se indispensável a atuação da enfermagem na assistência de qualidade e baseada nas melhores evidências científicas para prestar assistência a essas mulheres e suas famílias, visando a detecção precoce de fatores de risco e o melhor seguimento da gestação.

Em suma, este estudo se propôs a ampliar os conhecimentos sobre IA e PE, visto que, existe uma carência de pesquisas que relacionem as duas condições, sendo um campo de estudo ainda pouco explorado e com dados ainda incipientes em todo $\mathrm{o}$ mundo. De fato, esta investigação é pioneira no Brasil e destaca a necessidade de maiores publicações nessa área. Apesar das limitações, este estudo, traz dados que avançam no conhecimento e na tentativa de elucidar quais fatores estão relacionados ao desencadeamento PE, e a interferência da insegurança alimentar no seu desenvolvimento. Assim como serviu para instigar maiores investigações, sugere-se a realização de um estudo com gestantes oriundas do pré-natal de alto risco, a fim de traçar distintos perfis obstétricos

\section{CONCLUSÃO}

Diante do exposto, conclui-se que houve a associação entre a insegurança alimentar e nutricional e os fatores de risco para o desenvolvimento de PE em gestantes. Por causa disso é necessário que os profissionais de saúde atentem-se para a existência dessas condições durante o período gestacional. Esses dados são importantes para o direcionamento da assistência à saúde desse público. Deve-se estimular estudos que utilizem instrumentos como o QFA e a EBIA, pois a partir deles é possível identificar a insegurança alimentar em gestantes.

\section{REFERÊNCIAS}

1.Brasil. Ministério da Saúde. Política Nacional de Promoção da Saúde. Brasília: Ministério da Saúde. [Internet] 2018 [Acesso em 15 de Mai 2021]. Disponível em: http://bvsms.saude.gov.br/bvs/publicacoes/pol itica_nacional_promocao_saude.pdf.

2. Brasil. Lei ${ }^{\circ} 11.346$, de 15 de setembro de 2006. Cria o Sistema Nacional de Segurança Alimentar e Nutricional - SISAN com vistas em assegurar o direito humano à alimentação adequada e dá outras providências. Brasília: Ministério da Saúde. [Internet] 2006 [Acesso em 15 Mai 2021]. Disponível em: http://www.planalto.gov.br/ccivil_03/_Ato20 04-2006/2006/Lei/L11346.htm

3.Oliveira ACMD, Tavares MCM, Bezerra AR. Insegurança alimentar em gestantes da rede pública de saúde de uma capital do nordeste brasileiro. Ciênc. Saúde Colet. [Internet]. 2017 [Acesso em 15 Mai 2021]; 22 
(2):

519-26.

doi: https://doi.org/10.1590/141381232017222.27382015

4. American College of Obstetricians and Gynecologists. Task Force on Hypertension in Pregnancy. Obstet. gynecol. [Internet] 2013 [Acesso em 16 Mai 2021]; 122(5):1122-31. doi:10.1097/01.AOG.0000437382.03963.88.

5. Silva PLN, Oliveira JS, Santos APO, Vaz MDT. Prenatal and puerperal care of pregnant women at a health center of Minas Gerais regarding the risk of preeclampsia: clinical, nutritional and therapeutic aspects. J. Health Biol. Sci. [Internet] 2017 [Acesso em 16 Mai 2021]; 5(4):346-51. doi: 10.12662/23173076jhbs.v5i4.1222.

6. American College of Obstetricians and Gynecologists. Committee Opinion No. 638: first-trimester risk assessment for early-onset preeclampsia. Obstet. gynecol. [Internet] 2015 [Acesso em 16 Mai 2021]; 126(3):e25-7. doi: doi.org/10.1097/AOG.0000000000001049

7. Zata F, Nasir M. A Promising Effect of Pravastatin for Reducing Preeclampsia Incidence in High Risk Pregnant Women. IIMJ. [Internet] 2019 [Acesso em 16 Mai 2021]; 1(1):1-8. doi: 10.33086/iimj.v1i1.1385.

8. Say L, Chou D, Gemmill A, Tunçalp Ö, Moller A, Daniels J, et al. Global causes of maternal death: a WHO systematic analysis. Lancet Glob. Health. [Internet] 2014 [Acesso em 16 Mai 2021]; 2(6):e323-33. doi: 10.1016/S2214-109X(14)70227-X.

9. Henderson JT, Thompson JH, Burda BU, Cantor A, Beil T, Whitlock EP. Screening for Preeclampsia: A Systematic Evidence Review for the U.S. Preventive Services Task Force. JAMA. [Internet] 2017 [Acesso em 16 Mai 2021]; 317(16):166883. Disponível em: https://www.ncbi.nlm.nih.gov/books/NBK447 462/

10. O'Gorman N, Wright D, Poon LC, Rolnik DL. Multicenter screening for pre-eclampsia by maternal factors and biomarkers at 11-13 weeks' gestation: comparison with NICE guidelines and ACOG recommendations. Ultrasound Obstet
Gynecol. [Internet] 2017 [Acesso em 17 Mai 2021]; 49(6): 756-60. doi: https://doi.org/10.1002/uog.17455

11. Dietary Assessment Research Group. Resources. Food frequency questionnaire (FFQ) - Adults. [Internet] 2018 [Acesso em 17 Mai 2021]. Disponível em: http://gacusp.com.br/wp-

content/uploads/2020/01/QFA_Adulto.pdf.

12. Instituto Brasileiro de Geografia e Estatística (IBGE). Pesquisa Nacional por Amostra de Domicílios 2013 - Suplemento de Segurança Alimentar. Rio de Janeiro: IBGE. [Internet] 2014 [Acesso em 17 Mai 2021]. Disponível em: https://biblioteca.ibge.gov.br/visualizacao/livr os/liv91984.pdf

13. Demétrio F, Teles CADS, Santos DBD, Pereira M. A insegurança alimentar em mulheres grávidas está associada a determinantes sociais e desfechos nutricionais: uma revisão sistemática e metaanálise. Ciênc. Saúde Colet. [Internet] 2020 [Acesso em 17 Mai 2021]; 25(7), 2663-76. doi: https://doi.org/10.1590/141381232020257.24202018

14. Almeida JA, Santos AS, Nascimento MA, Oliveira JV, Silva DG, Mendes-Netto RS. Fatores associados ao risco de insegurança alimentare nutricional em famílias de assentamentos rurais. Ciênc. Saúde Colet. [Internet] 2017 [Acesso em 17 Mai 2021]; 22(2):479- 88. doi: 10.1590/141381232017222.27102015 .

15. Morais DC, Dutra LV, Franceschini SDCC, Priore SE. Insegurança alimentar e indicadores antropométricos, dietéticos e sociais em estudos brasileiros: uma revisão sistemática Ciênc. Saúde Colet. [Internet] 2014 [Acesso em 18 Mai 2021]; 19(5):14751488. doi: https://doi.org/10.1590/141381232014195.13012013

16. Ministério da Saúde (BR). Vigitel Brasil 2017: vigilância de fatores de risco e proteção para doenças crônicas por inquérito telefônico. Brasília: Ministério da Saúde. [Internet] 2018 [Acesso em 18 Mai 2021]. Disponível em: 
https://bvsms.saude.gov.br/bvs/publicacoes/vi gitel_brasil_2017_vigilancia_fatores_riscos.p df

17. Villamil, LAT, Rojas ACF, Pinzón OL. Cuidado nutricional en la prevención de la preeclampsia: Una revisión sistemática. Rev. esp. nutr. comunitaria. [Internet] 2018 [Acesso em 18 Mai 2021]; 24(2): 1-14. Disponível em: https://dialnet.unirioja.es/servlet/articulo?codi $\mathrm{go}_{\mathrm{o}}=6987984$

18. Hoseini, KS, Kazemi, F, Alimoradi Z, Oskoei, SSS, Alijani, H, Zolghadr S. Association between household food security and pregnancy complications. J. health soc. behav. [Internet] 2018 [Acesso em 18 Mai 2021]; 1(1): 26. doi: 10.4103/SHB.SHB_3_18

19. Oliveira AC, Santos AA, Bezerra AR, Barros AM, Tavares MC. Maternal Factors and Adverse Perinatal Outcomes in Women with Preeclampsia in Maceió, Alagoas. Arq Bras. Cardiol. [Internet] 2016 [Acesso em 18 Mai 2021]; 106(2):113-20. doi: 10.5935/abc.20150150.

20. Ferreira ETM, Moura NS, Gomes MLS, Silva EG, Guerreiro MGS, Oriá MOB. Maternal characteristics and risk factors for preeclampsia in pregnant women. Rev Rene. [Internet] 2019 [Acesso em 18 Mai 2021]; 20:e40327. doi: 10.15253/21756783.20192040327
Autor correspondente Mônica Oliveira Batista Oriá Rua Alexandre Baraúna, 1115 - Rodolfo Teófilo / Fortaleza-CE - CEP 60430-160 Email: profmonicaoria@gmail.com

Submissão: 2021-05-21

Aprovado: 2021-07-11 